\title{
L'évaluation participative des politiques de coopération au développement. Analyse comparée des dispositifs du Canada, de l'Union européenne et de la Banque mondiale
}

\author{
Steve Jacob, Nouhoun Diallo \& Pierre-Marc Daigneault \\ Université Laval
}

\section{Introduction}

Les politiques et programmes de coopération au développement ambitionnent d'atténuer, à défaut de résoudre complètement, les problèmes relatifs, entre autres, à la pauvreté, à la malnutrition et à l'analphabétisme qui frappent les pays du Sud. De nos jours, la gestion de l'aide à la coopération au développement est de plus en plus dominée par une ambition participative. Les bailleurs de fonds considèrent que cette façon de faire, accroît la transparence et la légitimité des actions qu'ils entreprennent auprès des populations locales. Dans cet article, nous nous intéressons à la manière dont l'évaluation des politiques et programmes de coopération au développement intègre ce paradigme participatif.

Face à une exigence de reddition de comptes et d'efficacité des interventions publiques, l'évaluation occupe une place importante dans la gestion de l'aide au développement international. Cet instrument de pilotage de l'action publique permet traditionnellement de formuler un jugement de valeur basé sur des informations méthodiquement recueillies et analysées. Audelà de cette dimension scientifique, l'évaluation présente également une dimension fortement politique qui permet aux décideurs d'avoir des données probantes pour justifier les décisions. Dans cette perspective, l'évaluation apparaît comme un enjeu de pouvoir que se disputent divers acteurs qui partagent des visions différentes de l'exercice.
Face à une exigence de reddition de comptes et d'efficacité des interventions publiques, l'évaluation occupe une place importante dans la gestion de l'aide au développement international.

L'histoire de l'évaluation de la coopération au développement reflète les évolutions qui caractérisent plus généralement les changements qui affectent la gestion publique. À ses origines, l'évaluation de la coopération était une entreprise essentiellement technocratique qui a même pu, au cours des années 1980, contribuer à des compressions budgétaires avant de mettre l'accent sur l'apprentissage, la participation et le développement des capacités locales.

L'histoire de l'évaluation de la coopération au développement reflète les évolutions qui caractérisent plus généralement les changements qui affectent la gestion publique.

En prenant en considération les multiples motivations et obstacles qui animent la réalisation d'une démarche évaluative, certains experts $^{1}$ pensent que les difficultés de l'évaluation sont liées à l'interaction entre les différents acteurs impliqués dans ce processus, à la légitimité de l'évaluateur et aux moyens de recueillir des informations fiables en vue de 
Les théoriciens de l'évaluation participative affirment que l'implication des parties prenantes à la démarche évaluative facilite une meilleure acceptabilité des résultats.

formuler des conclusions et des recommandations sur la gestion des interventions dans les domaines considérés. D'autres auteurs $^{2}$ considèrent que le traitement de ces difficultés doit faire l'objet d'un consensus ou, à tout le moins, d'une négociation entre les acteurs concernés. Ainsi, ils présentent l'évaluation participative comme " un autoexamen, une production de savoir collectif et une entreprise coopérative au sein desquels les parties prenantes à l'intervention d'aide au développement participent substantivement à l'identification des enjeux de l'évaluation, à la conception de la collecte et de l'analyse des données et aux suites à donner aux conclusions de l'évaluation » (traduction).

Plusieurs raisons ont incité les donateurs à associer les parties prenantes à leurs évaluations. Le premier motif énoncé en faveur de l'évaluation participative est d'ordre pragmatique $^{3}$. Cette approche est censée, d'une part, faciliter l'utilisation des résultats de l'évaluation et la gestion des programmes d'aide au développement et, d'autre part, contribuer à la résolution des problèmes et à la prise de décision politique. En ce qui concerne l'utilisation des résultats par les différentes parties prenantes, les théoriciens de l'évaluation participative affirment que l'implication des parties prenantes à la démarche évaluative facilite une meilleure acceptabilité des résultats et une plus grande appropriation des conclusions et des recommandations formulées lors de l'évaluation. Dans les projets de développement, ce sont ces parties prenantes ou du moins certaines d'entre elles qui auront ultimement à s'approprier et à mettre en œuvre les recommandations du rapport d'évaluation. En les intégrant dès le départ, l'évaluation prend déjà en considération leurs valeurs, leurs points de vue, leurs intérêts et leurs attentes à chacune des étapes du processus. À ce sujet, certains chercheurs ${ }^{4}$ estiment que l'absence des parties prenantes pourrait conduire à la constitution d'un contre-pouvoir à l'égard des décisions prises tandis qu'une évaluation qui encourage la participation minimiserait ces confrontations sur les résultats de l'évaluation. La participation des parties prenantes permet donc de désamorcer les champs de tension en cours de route, de rendre l'application des conclusions et des recommandations plus facile et d'accroître par le fait même le potentiel de réussite des programmes. Dans ces conditions, l'évaluation concourt ultimement à une amélioration de la situation des populations bénéficiaires par l'entremise des politiques et programmes de coopération au développement.

\section{Dans les projets de développement, ce sont ces parties prenantes ou du moins certaines d'entre elles qui auront ultimement à s'approprier et à mettre en ouvre les recommandations du rapport d'évaluation.}

Un second motif est d'ordre épistémologique: la participation permet de rehausser la qualité des connaissances et des jugements générés par l'évaluation. Partant de sa contribution à une décision politique, l'évaluation participative procure les informations essentielles à la prise de décisions. Les parties prenantes sont parmi les acteurs qui détiennent une partie importante de ces informations pertinentes. L'utilisation des informations provenant de ces sources multiples de perspectives et de connaissances, permet de produire une meilleure qualité du jugement de valeur et par conséquent d'aboutir à des résultats plus crédibles. Ces résultats ont un impact sur les attitudes et les actions des différents acteurs et peuvent conduire à des changements aux niveaux individuel, interpersonnel et collectif. 
Certains acteurs politiques se saisissent de ces résultats pour inscrire des problèmes à l'ordre $\mathrm{du}$ jour du gouvernement alors que d'autres s'en servent pour formuler des politiques et des stratégies, ce qui conduit à une prise de décisions plus rationnelles.

Le troisième motif du recours à l'évaluation participative est d'ordre politique. L'évaluation participative est faite ici dans un souci démocratique de participation citoyenne, d'habilitation et d'émancipation. Elle encourage la démocratisation de la connaissance et des changements sociaux en faveur des plus pauvres et des personnes démunies. Son application dans les pays en voie de développement vient en appui au discours de la démocratisation d'une part, et, d'autre part, elle répond au souci de tenir compte des conditions de la complexité et de la diversité sociale dans la résolution des problèmes publics. Dans l'évaluation des projets de développement, la participation crée non seulement un espace d'expression pour les citoyens, mais offre aussi les conditions dans lesquelles les débats doivent être menés pour aboutir à un consensus. Le contact avec les bénéficiaires conduit à une flexibilité et une adaptation dans la méthodologie des évaluateurs. En plus de son rôle de renforcement de la démocratie directe, l'évaluation joue aussi un rôle de justice sociale. En effet, la participation des parties prenantes fait de l'évaluation un lieu de communication, d'échange et une activité de négociation. Dans ce milieu de débat où chaque partie assiste de façon conjointe à la construction de l'offre et de la demande, la communauté parvient à répartir de façon plus équitable les pouvoirs ressortissants des domaines politique, économique et social.

Certaines mutations sociales ont renforcé le recours à l'évaluation participative; nous pouvons citer entre autres l'évolution de la politique d'aide au développement de la Banque mondiale, principal bailleur de fonds
Dans l'évaluation des projets de développement, la participation crée non seulement un espace d'expression pour les citoyens, mais offre aussi les conditions dans lesquelles les débats doivent être menés pour aboutir à un consensus.

Banque mondiale, principal bailleur de fonds des pays en voie de développement (PVD). En effet, depuis le consensus de Washington des années 1980, l'État a été rejeté en tant qu'acteur de développement économique. Les appuis budgétaires connaissaient un fléchissement en ce temps contrairement aux subventions et aux prêts qui faisaient légion. La gestion des projets de développement était fortement confiée à des organismes autonomes sous le contrôle des bailleurs de fonds ou à des gestionnaires privés. Les contrôles par les règles (contrôle financier, inspection, cour des comptes, etc.) devenaient insatisfaisants aux yeux des bailleurs de fonds compte tenu de leur rattachement à l'exécutif des pays bénéficiaires et des faiblesses liées à la lourdeur et à la lenteur administratives. Plusieurs changements ont contribué à réformer les modes de gestion des politiques et programmes de coopération. Il s'agit d'une part de la révision de cette politique de contrôle et de l'incorporation d'autres bailleurs de fonds dans un processus de coopération multilatérale fondée sur une logique de programmes et de résultats. D'autre part, au cours des années 1990, l'essor des concepts de gestion publique centrés autour de la «bonne » gouvernance, a modifié la place occupée par l'État dans le processus de développement et a transformé considérablement le système d'information des institutions aux fins d'améliorer les processus de gestion et les interactions entre l'État, les différents donateurs et les autres partenaires. Dans ce contexte, l'évaluation participative est présentée comme l'outil qui répond le mieux au souci de réduire l'exercice d'un pouvoir excessif par un seul acteur qui est bien souvent le bailleur de fonds. 
Au cours des années 1990, l'essor des concepts de gestion publique centrés autour de la « bonne » gouvernance, a modifié la place occupée par l'État dans le processus de développement.

À partir de 2004, une nouvelle tendance a pris place au niveau de l'aide au développement avec la déclaration de Paris. Cette déclaration voit le retour de l'aide budgétaire suivi des principes de gestion axée sur les résultats et de la responsabilité mutuelle. Ces principes exigent une collaboration entre les différents partenaires financiers, l'État bénéficiaire et les parties prenantes mettant ainsi en relief la participation de tous.

Malgré l'importance accordée à l'évaluation participative dans les projets de développement, l'interaction entre les différentes parties prenantes ne se fait pas sans difficulté. La relation entre le décideur, le bailleur de fonds (y comprise la rémunération de l'évaluateur) les gestionnaires du programme, le personnel de première ligne qui livre les services, les bénéficiaires du programme, la société civile et l'évaluateur fait l'objet d'un jeu complexe de coalition et de domination. L'évaluation participative peut être instrumentalisée dans ce cas pour répondre exclusivement aux besoins informationnels et d'imputabilité des organismes donateurs et renforcerait ainsi la relation asymétrique, voire unidirectionnelle, qui caractériserait l'évaluation de l'aide au développement $^{5}$. Cela dénierait à l'évaluation participative son rôle de prise en compte des besoins de toutes les parties prenantes et de bénéfices pour tous. En d'autres termes, il n'y a pas de relation nécessaire entre l'inclusion des parties prenantes et l'amélioration du sort des populations locales.

Dans cet article, nous souhaitons, d'une part, analyser cet écart entre le discours et l'action en matière d'évaluation de l'aide au développement et, d'autre part, commenter la distribution des coûts et des bénéfices de la participation entre les différentes parties prenantes. Trois questions guident la présente analyse: qu'est-ce que l'évaluation participative?; comment des institutions comme la Banque mondiale, l'Agence canadienne de développement international (ACDI) et l'Union européenne énoncent-elles l'évaluation participative dans leur guide?; et enfin, quels sont les avantages et inconvénients de l'évaluation participative du point de vue des parties prenantes des pays bénéficiaires de l'aide?

L'article est structuré autour de ces trois questions. La première partie est consacrée à la conceptualisation de l'évaluation participative. En nous basant sur cette conceptualisation, nous présentons et analysons dans la deuxième partie le discours sur l'évaluation participative de la Banque mondiale, de l'ACDI et de l'Union européenne à partir de leur guide. Dans la troisième partie du texte, nous discutons les difficultés de l'évaluation participative à travers l'écart entre la pratique et le discours. Nous passons également en revue les avantages et retombées positives de l'évaluation participative pour les communautés et les voies possibles d'amélioration. Cette dernière partie vise en effet à soumettre le discours dominant sur la participation à un « test de réalité » en le confrontant aux difficultés sous-jacentes à la participation et à proposer des pistes d'explication quant à l'embrassement plus ou moins sincère de l'évaluation participative par les organisations étudiées. 


\section{Conceptualisation de l'évaluation participative}

Plusieurs expressions sont aujourd'hui employées pour désigner l'évaluation participative et ses différentes variantes : stakeholder approach beneficiary assessment and community assessment, auto-évaluation, évaluation de quatrième génération, évaluation centrée sur l'utilisation, realistic evaluation, évaluation démocratique, évaluation émancipatrice. Derrière ces nombreuses définitions se cache la faiblesse conceptuelle de l'évaluation participative qui n'épargne pas le domaine de la coopération au développement ${ }^{6}$.

\section{La grille conceptuelle d'analyse}

La grille conceptuelle retenue dans le cadre de la présente analyse des guides des bailleurs de fonds est celle développée par Daigneault et $\mathrm{Jacob}^{7}$ qui constitue une extension du cadre conceptuel de Cousins et Whitmore ${ }^{3}$.

L'évaluation participative est caractérisée par la collaboration des évaluateurs lors de l'évaluation avec les individus, les groupes ou les communautés qui ont un intérêt dans le programme. Deux courants d'évaluation participative sont identifiés par les chercheurs en évaluation: le courant pratique (practical participatory evaluation ou P-PE) et le courant transformatif «tranformative participatory evaluation » (T-PE) ${ }^{3}$. La différence entre les deux se situe au niveau de leur visée. Les trois dimensions constitutives de l'évaluation participative sont la diversité des parties prenantes impliquées dans l'évaluation, l'étendue de leur implication et le partage du contrôle relatif aux décisions techniques ${ }^{7}$.

La première dimension, la diversité des parties prenantes, comme son nom l'indique, a trait à la variété des participants à l'évaluation. Elle mesure l'inclusion de divers types de parties prenantes non évaluatives (c.-à-d. autres que l'évaluateur) au processus évaluatif, soit les (1) décideurs et concepteurs de politiques (gestionnaires et élus); les (2) opérateurs de mise en œuvre et employés de première ligne responsables de la livraison des services; (3) les bénéficiaires directs et indirects du programme; les bénéficiaires potentiels et les personnes lésées par le programme; et finalement (4) la société civile et les citoyens. La seconde dimension, l'étendue de la participation, réfère à l'association des parties prenantes non évaluatives à différentes étapes du processus évaluatif: (1) définition des enjeux, questions et critères d'évaluation et la conception du devis d'évaluation; (2) la collecte et analyse des données; (3) formulation des conclusions et des recommandations; et (4) la diffusion des conclusions de l'évaluation. Elle est mesurée en fonction du nombre d'étapes ou les parties prenantes non traditionnelles sont impliquées. Quant au contrôle des décisions techniques, il réfère à la répartition du pouvoir décisionnel entre les différents acteurs impliqués dans la démarche. Cette dimension est rangée sur une échelle allant des décisions contrôlées exclusivement par l'évaluateur aux décisions prises exclusivement par les autres parties prenantes.

Les trois dimensions constitutives de l'évaluation participative sont la diversité des parties prenantes impliquées dans

l'évaluation, l'étendue de leur implication et le partage du contrôle relatif aux décisions techniques.

Pour chacune de ces dimensions, il est possible d'établir des seuils précis permettant de distinguer si une évaluation est participative ou non. Dans le cas de la diversité des parties prenantes, nous postulons qu'une évaluation est participative dès qu'elle inclut au moins un type de partie prenante outre l'évaluateur. En effet, la participation implique d'aller au-delà de l'inclusion des acteurs non évaluatifs de l'évaluation. Dans le cas de l'étendue de 
l'implication, une évaluation est jugée participative si les parties prenantes sont associées à au moins une étape du processus. En ce qui concerne le contrôle, les parties prenantes non évaluatives doivent minimalement avoir une influence indirecte sur les décisions techniques, par exemple suite à leur consultation par l'évaluateur. Le rôle des parties prenantes doit aller au-delà de celui de «source de données». Le contrôle peut être exercé formellement (p. ex., autorité légale) ou informellement (persuasion, compétences méthodologiques et sociales). Dans tous les cas, il est important de souligner que ces seuils constituent des bornes inférieures. Une évaluation sera d'autant plus participative dans la mesure où elle associe une grande diversité de parties prenantes à plusieurs étapes du processus et que ces acteurs ont le dernier mot en ce qui a trait à la prise de décision.

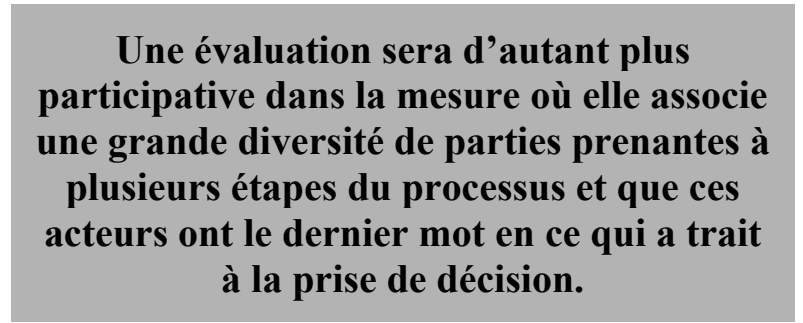

Grille conceptuelle d'analyse

\begin{tabular}{|c|c|}
\hline Dimension & Évaluation participative \\
\hline \multirow{2}{*}{$\begin{array}{l}\text { Diversité des parties } \\
\text { prenantes non évaluatives }\end{array}$} & Au moins un type de partie prenante parmi : \\
\hline & $\begin{array}{l}\text { 1. Décideurs et concepteurs des politiques } \\
\text { 2. Opérateurs de mise en œuvre, employés de première ligne. } \\
\text { 3. Bénéficiaires directs, indirects et potentiels du programme et } \\
\text { personnes lésées par le programme } \\
\text { 4. Société civile et citoyens. }\end{array}$ \\
\hline \multirow[t]{2}{*}{ Étendue de l'implication } & $\begin{array}{l}\text { Association des parties prenantes au processus évaluatif à au moins } \\
\text { une étape parmi : }\end{array}$ \\
\hline & $\begin{array}{l}\text { 1. Définition des enjeux et critères d'évaluation ; conception du devis } \\
\text { d'évaluation. } \\
\text { 2. Collecte et analyse des données. } \\
\text { 3. Formulation des conclusions et des recommandations. } \\
\text { 4. Diffusion des conclusions de l'évaluation. }\end{array}$ \\
\hline $\begin{array}{l}\text { Contrôle des décisions } \\
\text { techniques }\end{array}$ & $\begin{array}{l}\text { Les parties prenantes ont minimalement une influence sur les } \\
\text { décisions. Ils ne sont pas uniquement pas des « sources de données » } \\
\text { mais influencent réellement l'évaluation. }\end{array}$ \\
\hline
\end{tabular}

\section{Les ambitions participatives de trois organisations d'aide au développement}

Dans cette section, nous tentons de déterminer si les ambitions affichées pour les évaluations commanditées par les institutions d'aide au développement sont réellement participatives. Nous allons dans ce cadre apprécier les attentes fondées sur l'évaluation participative par trois bailleurs de fonds parmi les plus en vue, à savoir une organisation internationale, la Banque mondiale (BM), une organisation supranationale l'Union européenne (UE), et une organisation nationale, soit l'Agence canadienne de développement international (ACDI). Nous confrontons la grille d'analyse présentée à la section précédente aux guides 
d'évaluation des trois organismes retenus. Nous nous attardons sur les guides d'évaluation, car ces derniers sont censés exprimer la vision organisationnelle de l'évaluation et présenter la manière dont la pratique évaluative devrait idéalement être mise en œuvre sur le terrain. En somme, les guides articulent le discours « officiel» de ces organismes sur l'évaluation. Ils permettent d'harmoniser les pratiques des évaluateurs qui collaborent avec ces organismes à travers le monde et envoient un signal clair sur la marche à suivre en la matière.

\section{L'évaluation à la Banque mondiale}

La Banque mondiale est le principal bailleur de fonds des projets de développement. Il s'agit d'une institution internationale de 185 pays qui a pour objectif de réduire la pauvreté dans le monde à travers des programmes dont le financement est essentiellement constitué de prêts ou de subventions remboursables. À la fin des années 1990, la Banque mondiale a amorcé un virage participatif et elle a mis l'accent sur le travail en partenariat avec les donateurs, les clients et les organisations qui composent la Banque. Au même moment, la participation est devenue un élément central de sa politique générale et de l'évaluation en particulier. Dans ce cas, elle implique les différentes directions de la Banque, les gouvernements et la société civile dans sa démarche qui vise à prendre en considération le point de vue des partenaires, des clients, du personnel et de la société civile dans les évaluations réalisées. Au-delà de cette intention générale, il est utile de chercher à savoir si la prétention participative de l'évaluation de la Banque mondiale correspond aux critères établis. En d'autres termes, au-delà du discours, cette organisation promeut-elle une conception véritablement participative de l'évaluation?

En ce qui concerne la diversité des parties prenantes, les différents types d'évaluation participative évoqués par les publications qui encadrent l'évaluation à la Banque mondiale en mentionnent l'importance. Ces documents font ainsi référence à la population locale dans l'évaluation participative rurale, aux bénéficiaires des projets dans l'évaluation par les bénéficiaires et à l'ensemble des parties prenantes dans l'évaluation et le suivi participatif. Malgré cette référence à l'ensemble des parties prenantes, la Banque mondiale ne fait pas explicitement mention des bénéficiaires indirects ou des personnes lésées dans son guide. Cela nous amène à présumer que la diversité des parties prenantes est limitée aux cercles proches de la gestion du projet sans chercher à impliquer systématiquement une grande variété de participants à l'évaluation.

Au niveau de l'étendue de la participation, les guides de la Banque mondiale accordent une place importante aux parties prenantes dans les tâches qui structurent le processus évaluatif. L'implication s'étend à différentes tâches telles que l'identification des problèmes, la collecte des données, l'analyse et l'interprétation des données et la formulation des recommandations ${ }^{8}$. Les guides restent par contre muets sur la sous-étape de la conception du devis d'évaluation. Cette omission suggère que les participants peuvent possiblement être soumis à l'influence d'une méthode de participation prédéfinie sans avoir l'occasion de s'exprimer à ce sujet ni de disposer d'un espace de discussion voire de contestation des enjeux méthodologiques qui structurent l'ensemble de la démarche.

En ce qui concerne le contrôle des décisions techniques, les documents servant de guide à la réalisation d'une évaluation nous informent peu, voire pas du tout, sur les éléments relatifs à son déclenchement et à la décision d'initier une évaluation. Cependant, cette phase est rarement participative puisque c'est le groupe d'évaluation indépendant (GEI), qui relève du conseil d'administration de la Banque, qui détermine annuellement les évaluations à 
entreprendre et leur nature en fonction des objectifs qui sont poursuivis. Les gestionnaires internes de l'évaluation sont donc les principaux responsables de cette pré-étape même s'il leur arrive de tenir compte des partenaires comme le confirme la déclaration qui stipule que «les méthodes participatives permettent d'associer activement à la prise de décision les parties prenantes au projet, programme ou stratégie et de les amener à souscrire pleinement aux résultats et recommandations $\rangle^{8}$. Cette prise en compte se fait souvent après la validation des méthodes de travail, au moment de la collecte des données, des discussions sur les résultats préliminaires et des recommandations. La prise de décisions relatives à la définition des enjeux, des questions et critères d'évaluation peut faire l'objet de discussions, mais relève essentiellement des évaluateurs. En résumé, les parties prenantes sont impliquées dans quelques étapes, mais sans disposer de pouvoir décisionnel.

L'évaluation à la Banque mondiale satisfait aux critères minimaux en matière de diversité des parties prenantes et d'étendue de l'implication. Cela n'est pas le cas pour la troisième dimension, soit le contrôle des décisions techniques, qui est sousdéveloppée dans les guides.

Au regard de ce qui précède, l'évaluation à la Banque mondiale satisfait aux critères minimaux en matière de diversité des parties prenantes et d'étendue de l'implication. Cela n'est pas le cas pour la troisième dimension, soit le contrôle des décisions techniques, qui est sous-développée dans les guides. De deux choses l'une : soit, le contrôle décisionnel est effectivement partagé, mais qu'il n'en est pas fait mention dans le guide, soit ce silence indique que celui-ci n'est pas partagé. Nous penchons en faveur de la deuxième hypothèse: en ne prêtant pas une grande attention aux éléments de pouvoir, nous présumons que la Banque mondiale ne les considère pas comme prioritaires. En effet, si l'évaluation devait impliquer davantage les parties prenantes, le guide, qui est, rappelonsle, un message envoyé aux évaluateurs qui travaillent pour le compte de la Banque mondiale, serait plus explicite à ce sujet et y accorderait une place plus grande. Dans ces conditions, le discours de la Banque mondiale dépeint une évaluation de nature technocratique où l'évaluateur agit davantage comme un inspecteur faisant faiblement participer les parties prenantes en matière de contrôle décisionnel que comme un facilitateur.

Le discours de la Banque mondiale dépeint une évaluation de nature technocratique où l'évaluateur agit davantage comme un inspecteur faisant faiblement participer les parties prenantes en matière de contrôle décisionnel que comme un facilitateur.

\section{L'évaluation à l'Agence canadienne de développement international}

Contrairement à la Banque mondiale qui est une institution bancaire multinationale, l'Agence canadienne de développement international (ACDI) est une entité qui relève du gouvernement fédéral canadien. Elle est le «... principal organisme responsable de l'aide au développement. Elle a pour mandat d'appuyer les activités de développement durable dans les pays en développement afin de réduire la pauvreté et de rendre le monde plus sûr, plus juste et plus prospère $\iota^{9}$. Pour l'ACDI, la participation des principaux intéressés est indispensable à l'atteinte des résultats et elle est considérée comme un facteur clé de la réussite et de l'amélioration continue des étapes de la conception, de l'exécution, de la surveillance et de l'évaluation de l'investissement aux projets. En ce qui concerne l'évaluation, la participation est mobilisée dans une orientation plus pragmatique et offre des perspectives de 
viabilité à long terme. À la lumière des critères de participation retenus, nous pouvons tirer plusieurs enseignements de la nature participative de l'évaluation de cette organisation.

Pour l'ACDI, la participation des principaux intéressés est indispensable à l'atteinte des résultats et elle est considérée comme un facteur clé de la réussite et de l'amélioration continue des étapes de la conception, de l'exécution, de la surveillance et de l'évaluation de l'investissement aux projets.

$\mathrm{Au}$ niveau de la diversité des parties prenantes, le guide distingue plusieurs catégories d'acteurs pouvant être associés à l'évaluation. Ainsi, «... l'évaluateur pourra consulter les représentants du gouvernement bénéficiaire, les bénéficiaires, les organisations de la société civile et organisations non gouvernementales, les agents de projet, personnels et gestionnaires de directions générales de programme à l'ACDI...» ${ }^{9}$ (les italiques sont de nous). Quant à l'étendue de la participation des non-évaluateurs, les principaux intéressés, y compris les membres de la société civile, peuvent participer directement à l'établissement des paramètres de mesure des résultats, à la sélection des parties prenantes à impliquer dans l'évaluation, à la sélection des méthodes, à la recherche de solutions pour régler les problèmes qui surgissent, au processus évaluatif proprement dit et à la communication des résultats ${ }^{9}$. Elle mentionne en outre que les acteurs intéressés aux projets doivent être prêts à consacrer le temps et l'énergie nécessaires à leur participation à l'évaluation. Le rôle qui leur est assigné consiste d'une part à examiner les enjeux, les développements et les résultats de l'évaluation et d'autre part de mettre à profit leur connaissance particulière des réalités locales et leur savoir-faire pour améliorer la situation. Les moyens utilisés pour intéresser les bénéficiaires sont décrits dans le guide ${ }^{9}$ et suivent un cheminement cohérent. Il s'agit pour le gestionnaire de premièrement prendre contact avec les éventuels groupes intéressés (bénéficiaires, administrations publiques locales, organisations communautaires locales, ONG, autres donateurs, médias) et d'examiner leur rapport avec le projet, leurs intérêts et la forme que pourrait prendre leur contribution. Deuxièmement, il amorce le dialogue et troisièmement, il voit avec les intéressés s'ils veulent y participer afin de discuter de leur possible contribution. Le guide de l'ACDI mentionne explicitement que les principaux intéressés peuvent participer directement à l'établissement des paramètres de mesure des résultats et des rapports ${ }^{9}$. Enfin, l'évaluateur incorpore la stratégie de participation des intéressés à son plan de travail. Même si l'initiative d'entamer une évaluation relève de

Même si l'initiative d'entamer une évaluation relève de l'ACDI qui détient le dernier mot en la matière, le contrôle des décisions techniques est davantage partagé avec les parties prenantes dans le cas canadien que dans celui présenté à la Banque mondiale.

l'ACDI qui détient le dernier mot en la matière, le contrôle des décisions techniques semble davantage partagé avec les parties prenantes que dans le cas de la Banque mondiale. Il apparaît donc que la façon dont on choisit de procéder à l'évaluation au sein de cette agence est fonction des attentes de l'ACDI ou des partenaires intéressés, du contexte local et de ses éventuelles contraintes et des capacités des principaux intéressés ${ }^{9}$.

Dans le cas des évaluations de l'ACDI, les différents acteurs qui sont associés à la démarche peuvent intervenir sur des enjeux très variés tels que l'objet, le calendrier, le 
déroulement (méthode, analyse et formulation des conclusions et recommandations) et la communication des résultats de l'évaluation. À l'aune de ces différents éléments contenus dans les guides, il apparaît que l'évaluation de l'ACDI s'inscrit dans une démarche participative. La diversité des parties prenantes semble élevée et il semble y avoir un réel partage du contrôle décisionnel.

L'évaluation de l'ACDI s'inscrit dans une démarche participative. La diversité des parties prenantes semble élevée et il semble y avoir un réel partage du contrôle décisionnel.

\section{L'évaluation de l'Union européenne}

Au niveau de l'Union européenne, l'office de coopération EuropeAid de la Commission gère les programmes communautaires et vise à améliorer l'efficacité de l'aide extérieure. Pour y parvenir, cette institution estime que $«$... la société civile, les organisations internationales et les gouvernements des États membres de l'Union européenne sont tous des acteurs importants $»^{10}$. Il apparait donc que la gestion des politiques et programmes de coopération au développement au sein de l'Union européenne diffère sur le plan politique et économique des deux situations précédentes. Sur le plan politique, elle est constituée d'un ensemble de pays avec des visions politiques différentes qu'il convient de fédérer autour de principes et d'objectifs partagés. À ce sujet, depuis le début des années 1990, le Traité de Maastricht précise que la politique européenne de coopération au développement doit être complémentaire à celle menée par les États membres. Sur le plan économique, l'Union européenne tire ses ressources de la cotisation de ces pays. De plus, contrairement à la Banque mondiale ou à l'ACDI, où l'évaluation n'est pas à ses débuts, elle semble être plus récente au niveau de l'Union européenne. La pratique évaluative a toutefois déjà connu une grande évolution ces dernières années du fait de la transformation du cadre institutionnel dans lequel s'exerce l'aide internationale et la tendance générale vers une gestion plus orientée vers les résultats ${ }^{11}$. À l'intérieur du guide de l'Union européenne, la pratique de l'évaluation participative en tant qu'approche d'évaluation n'est pas mentionnée explicitement.

À défaut d'être détaillée, la diversité des parties prenantes est signalée dans le guide. Ce document énumère les parties prenantes suivantes: les individus, les groupes ou les organisations qui ont des intérêts directs ou indirects dans l'intervention évaluée et dans l'évaluation elle-même. Cette énumération est suffisamment floue pour permettre d'associer une variété d'acteurs à la démarche évaluative. Nous présumons donc que la diversité est potentiellement élevée.

L'étendue de la participation semble assez large. L'implication des parties prenantes s'étend des premières étapes du processus alors que des consultations menées par l'équipe d'évaluation auprès des participants à la discussion $\mathrm{du}$ rapport d'évaluation provisoire lors d'un séminaire élargi. Durant le processus d'évaluation, des ateliers participatifs, des groupes de discussion ou des panels d'experts sont organisés. Le guide prévoit en outre que les pays bénéficiaires peuvent être invités à discuter des constats et des recommandations de l'évaluation.

Le contrôle des décisions techniques n'est pas clairement mentionné dans le guide et semble donc être exclusivement du ressort du bailleur de fonds et de l'évaluateur. Ce contrôle de l'évaluation semble exclusivement entre les mains de la Commission européenne. La prise de décision n'implique aucune représentation formelle ni des bénéficiaires ni même des 
acteurs non membres de la Commission ${ }^{11}$. La Commission européenne reste le commanditaire et le principal responsable de l'évaluation du point de vue décisionnel. En somme, à l'instar de la Banque mondiale, le critère de contrôle décisionnel de l'évaluation par les parties prenantes non traditionnelles ne semble pas atteint dans les évaluations de l'Union européenne.

Ce contrôle de l'évaluation semble exclusivement entre les mains de la Commission européenne. La prise de décision n'implique aucune représentation formelle ni des bénéficiaires ni même des acteurs non membres de la Commission.

\section{La rhétorique participative des bailleurs de} fonds de la coopération au développement

Au regard de ce qui est mentionné dans les différents guides, il est clair que les trois bailleurs de fonds cherchent à impliquer, à des degrés variables, les parties prenantes à la réussite de l'aide au développement et de son évaluation mais sans toutefois que l'évaluation participative soit toujours mentionnée explicitement. Qui plus est, les guides traitant explicitement de la participation ne semblent pas prescrire de manière très rigide ou obliger celle-ci; ils se contentent plutôt de la permettre. Les niveaux d'implication dans les différents processus demeurent cependant hétérogènes et, en outre, il faut souligner qu'implication n'égale pas nécessairement participation.

L'ACDI semble accorder une attention plus grande au paradigme participatif dans l'évaluation des actions qu'elle entreprend dans le cadre de la coopération au développement en comparaison avec la Banque mondiale et l'Union européenne où l'évaluation se présente comme moins participative qu'elle n'y paraît à première vue.
L'implication des parties prenantes au niveau de l'ACDI est peut-être facilitée par sa structure moins hiérarchique. Quant à la Banque mondiale, sa hiérarchie et son statut apparaissent comme des lourdeurs et des entraves qui ne facilitent pas cette implication et le partage des décisions. En tant qu'institution bancaire, elle doit faire des profits et en tant qu'entreprise, elle doit rendre compte à des associés qui poursuivent des objectifs différents et qui ont des points de vue qui ne convergent pas toujours. Par conséquent, elle rend des comptes d'abord aux porteurs de parts («shareholders») et seulement, ensuite aux parties prenantes («stakeholders»). Ce statut repositionne le rôle des évaluateurs qui sont tournés vers l'analyse coût-bénéfice et l'analyse des performances qui requièrent moins l'implication des parties prenantes. En ce qui concerne l'Union européenne, sa structure hiérarchique joue aussi en défaveur de la participation, car elle représente une supra structure politique et économique qui doit rendre compte à la Commission européenne et aux gouvernements des États membres. En conclusion, nous observons que la hiérarchie organisationnelle, les statuts et le type de coopération semblent influencer la nature de l'évaluation participative des institutions. Cette hypothèse devra cependant être vérifiée. Nous consacrerons la dernière partie de cet article à un " test de réalité » de la participation qui consiste en une présentation des avantages et inconvénients de l'évaluation participative du point de vue des parties prenantes.

L'ACDI semble accorder une attention plus grande au paradigme participatif dans l'évaluation des actions qu'elle entreprend dans le cadre de la coopération au développement en comparaison avec la Banque mondiale et l'Union européenne. 


\section{L'évaluation participative au concret : difficultés, avantages et perspectives}

Comme nous venons de le voir, l'évaluation telle que présentée dans les guides des trois organismes étudiés ne répond pas, exception faite de l'ACDI, à tous les critères retenus pour qualifier celle-ci de participative (en considérant l'omission de la participation ou de l'une de ses dimensions dans un guide comme un indicateur de son absence des pratiques). Dans notre analyse, nous avons constaté que le discours sur l'évaluation participative pèche surtout sur la dimension de contrôle des décisions techniques. Dans cette dernière partie de l'article, nous voulons nous arrêter sur l'écart entre le discours et l'action en matière d'évaluation participative. Nous avons vu qu'il existe un décalage entre le discours général relatif à l'idéal participatif de l'aide au développement et sa traduction dans les guides d'évaluation. Partant de là, nous cherchons à savoir dans quelle mesure les difficultés de l'évaluation participative qui sont présentées par les théoriciens de l'évaluation peuvent constituer des pistes d'explication qui permettraient de comprendre la faible implication des parties prenantes selon le portrait tracé par les guides des bailleurs de fonds de la coopération au développement. En généralisant ce propos, il est également possible d'améliorer les retombées de l'évaluation participative pour les parties prenantes locales qui doivent être conscientes que cette démarche n'est pas exempte de difficultés et d'embûches.

\section{Les difficultés de l'évaluation participative}

Les difficultés dans la réalisation d'une évaluation participative de l'aide au développement sont de plusieurs ordres. Nous les regroupons en quatre catégories. Primo, le facteur le plus important semble être l'existence d'un fossé culturel entre les différents acteurs impliqués dans le processus évaluatif. En effet, les évaluateurs sont souvent formés dans un contexte fort différent de celui des bénéficiaires des politiques et programmes de coopération au développement. Il existe donc des écarts culturels qui peuvent entraver le travail des évaluateurs ou empêcher les bénéficiaires d'agir. Il est donc nécessaire de prévoir une évolution pour s'assurer que l'évaluation de l'aide ne soit pas réalisée avec les seules préoccupations de la société américaine, canadienne ou européenne. À cet égard, un plus grand contrôle des décisions relatives à l'évaluation par les parties prenantes non évaluatives permettrait probablement de pallier ce problème. Pour que cela soit effectif, il sera nécessaire de prendre en considération les attitudes des acteurs impliqués dans l'évaluation et de tenir compte de la déférence de certains envers les élites qui pourraient annihiler les potentialités de la participation.

Secundo, la participation présente en ellemême quelques dilemmes identifiés, entre autres, par les chercheurs qui s'intéressent au processus politique délibératif. Il s'agit principalement du type de représentativité (statistique ou politique), de l'égalité entre les parties prenantes et de la possible instrumentalisation de la démarche par un groupe particulier qui domine le processus. Tous ces éléments peuvent in fine affecter l'efficacité des décisions prises et retarder l'ensemble de la démarche évaluative. Compte tenu des enjeux soulevés par ces remarques, il semble évident que l'évaluation participative ne peut faire l'économie d'une réflexion sur ces questions et qu'il importe de s'interroger sur la nature de l'implication des parties prenantes à l'évaluation en s'assurant de doter les participants du bagage social et cognitif nécessaire à l'accomplissement de leurs tâches.

Tertio, la participation exige souvent plus de temps et de ressources que l'évaluation traditionnelle. En effet, le nombre d'acteurs 
impliqués complexifie le processus de recherche et de décision.

\section{L'évaluation participative vise à sortir de la logique où les communautés bénéficiaires et la société civile demeurent soumises aux pressions des autorités de leur pays et à l'expertise de l'évaluateur.}

Enfin, le partage du pouvoir est l'élément essentiel, mais sensible et même parfois problématique pour le contrôle des décisions. Dans une évaluation conventionnelle, chaque participant use des moyens dont il dispose pour contrôler les décisions et aucun acteur ne souhaite perdre une parcelle de ses prérogatives. Ce comportement pose moins de problèmes étant donné le nombre limité de participants. Les décideurs utilisent leur autorité pour commander et imposer leur point de vue, le bailleur de fonds utilise son pouvoir de financement et influence les bénéficiaires et l'évaluateur. Quant à l'évaluateur et au gestionnaire, ils utilisent la connaissance pour faire valoir leur point de vue et, dans certains cas, tenter de l'imposer aux autres. Dans ce jeu de coalition et de domination, les vrais bénéficiaires et la société civile apparaissent désavantagés puisqu'ils ne peuvent pas lutter à force égale avec les autres acteurs impliqués dans une démarche évaluative. Le contrôle des décisions techniques ne semble pas épuiser le sens du pouvoir. D'autres dimensions telles que le statut social, l'expertise et la capacité sociale à s'exprimer et à persuader entrent également en jeu. L'évaluation participative vise à sortir de la logique où les communautés bénéficiaires et la société civile demeurent soumises aux pressions des autorités de leur pays et à l'expertise de l'évaluateur. Le niveau d'implication des parties prenantes dépendra de l'ouverture et de la sensibilité de ceux qui détiennent le pouvoir.
Une mauvaise évaluation des bénéfices de la participation par les organisations d'aide au développement pourrait également expliquer sa faible prise en compte. Or, ces bénéfices sont significatifs, en particulier pour les participants.

\section{Les avantages pour les parties prenantes}

La participation est très importante pour les parties prenantes dans le cadre des programmes de coopération au développement. Elle répond en effet à un besoin éthique au nom duquel chaque personne commande son propre sort. Ces politiques, programmes ou projets sont souvent initiés par les gestionnaires et les gouvernements sans une connaissance réelle et spécifique des besoins des principaux intéressés. Comme le souligne les consultants de la Banque mondiale, seul le pauvre est capable de définir ce qu'est sa pauvreté et de proposer les perspectives pour s'en sortir.

Une fois le projet mis en œuvre, ce sont les activités décrites qui sont exécutées. Malgré les standards de performance mis en place par ces organismes et les experts, certains éléments sont hors du contrôle et peuvent être à la base des échecs d'un projet ou d'un programme. L'évaluation constitue donc le moment à partir duquel on s'interroge sur les actions entreprises et sur la réalisation des activités, l'atteinte des objectifs ou des impacts sur le bien-être de la communauté. Or, c'est pendant cette période que des propositions peuvent être suggérées en vue de réorienter les activités en direction d'impacts attendus.

La participation des bénéficiaires et d'autres acteurs non évaluatifs permet alors de mesurer la contribution du projet à la résolution de leurs problèmes par le développement de méthode et la constitution éventuelle d'indicateurs permettant de mesurer les besoins spécifiques des populations locales. 
La participation des bénéficiaires et d'autres acteurs non évaluatifs permet alors de mesurer la contribution du projet à la résolution de leurs problèmes par le développement de méthode et la constitution éventuelle d'indicateurs permettant de mesurer les besoins spécifiques des populations locales. Dans cette perspective, l'évaluation participative favorise également davantage le suivi des recommandations et la mise en place de changements opérationnels. En somme, la participation à l'évaluation permet aux parties prenantes de prendre en main leurs destinées et d'initier des changements qui répondent à leurs besoins puisqu'en étant associées aux différentes étapes de la démarche évaluative, elles ont la possibilité de corriger les incompréhensions qui surviennent en amont et réviser en aval les conclusions et recommandations mal fondées ou biaisées qui pourraient mener à des décisions sous-optimales.

Un autre aspect bénéfique de la participation pour les parties prenantes est l'apprentissage des acteurs et le renforcement des compétences. Que ce soit à la Banque mondiale

\section{Conclusion}

Théoriquement, l'évaluation participative des politiques et programmes de coopération au développement semble répondre adéquatement aux attentes des différents acteurs impliqués dans ce domaine. Nous avons vu que cette pratique présente différents avantages dès lors que les acteurs prennent conscience des difficultés à surmonter. C'est sans doute pour cette raison que le discours participatif des bailleurs de fonds tend à se généraliser pour toutes les étapes du cycle de vie des politiques, des programmes ou des projets qu'ils promeuvent.

Toutefois, en analysant les spécifications contenues dans les guides d'évaluation de la Banque mondiale, de l'Union européenne et ou à l'ACDI, les différents acteurs sélectionnés sont formés avant de participer au processus d'évaluation. La politique des deux institutions en matière de suivi évaluatif encourage la formation des évaluateurs locaux pour faire tout d'abord l'auto-évaluation des projets. Une telle politique est avantageuse à double titre pour la population car, d'une part, elle bénéficie d'un personnel local compétent et moins onéreux que les consultants internationaux et, d'autre part, ceux qui participent à l'évaluation sont mieux formés et il reste un capital d'expérience pour le développement local. Sur le plan individuel, la participation permet non seulement de motiver les acteurs, mais aussi d'acquérir des compétences sociales, politiques et techniques utiles au développement de leur communauté.

\section{Un autre aspect bénéfique de la} participation pour les parties prenantes est l'apprentissage des acteurs et le renforcement des compétences. de l'Agence canadienne de développement international, nous constatons que ces principes se conçoivent différemment. En effet, en confrontant les guides à la grille proposée, des résultats divergents selon les institutions apparaissent. Cette analyse comparée met en évidence le fait que les bailleurs de fonds qualifient d'évaluation participative des réalités très différentes et qu'ils ne poursuivent pas tous les mêmes objectifs en la matière. Ainsi, l'évaluation de l'ACDI est davantage orientée vers la participation que celles de la Banque mondiale et de l'Union européenne. Dans les deux derniers cas, nous constatons que les bailleurs de fonds sont réticents à partager les éléments relatifs au contrôle des décisions techniques. Il semble 
donc que le principe d'ouverture se heurte à la crainte de la perte de contrôle sur la démarche ou, à tout le moins, que le partage du contrôle ne soit pas conçu comme nécessaire à l'évaluation participative. Ceci limite bien entendu la portée de la participation et les capacités d'apprentissage des communautés locales. Actuellement, la plupart des évaluations sont réalisées dans un cadre (centralisé, multilatéral, axé sur le contrôle et la reddition des comptes internes) qui ne facilite pas un véritable partage du pouvoir. C'est seulement lorsque ces réticences seront levées que l'évaluation des politiques et programmes de coopération au développement s'engagera véritablement dans une direction orientée vers la participation.

\section{L'évaluation de l'ACDI est davantage} orientée vers la participation que celles de la Banque mondiale et de l'Union européenne. Dans les deux derniers cas, nous constatons que les bailleurs de fonds sont réticents à partager les éléments relatifs au contrôle des décisions techniques.

Malgré ces critiques, il faut noter que l'évaluation participative des institutions étudiées a connu de grandes évolutions. La Banque mondiale qui est pionnière dans le domaine, a commencé, en 1986, par des formations à l'auto-évaluation des projets.

\section{Remerciements}

Les auteurs remercient l'École de la fonction publique du Canada pour l'aide financière accordée à ce projet de recherche. Les opinions exprimées dans ce document ne sont pas nécessairement celles de l'École de la fonction publique du Canada ou du gouvernement du Canada. Ce projet de recherche a également été rendu possible grâce au soutien financier du Fonds québécois de recherche sur la société et la culture (FQRSC).

\section{Bibliographie}

1 Gendreau, F., Kikhela, D. N. et V. Guérin (dirs.) (1998). L'évaluation des politiques et des programmes de population. Paris : John Libbey Eurotext / Aupelf UREF.
L'évaluation participative ne se résume pas seulement à cet enjeu du développement des capacités évaluatives, mais il constitue un moyen de développer, au sein des pays bénéficiaires, des compétences qui pourront être mobilisées dans une démarche d'évaluation participative. C'est dans ce contexte que l'évaluation participative s'est intensifiée à la fin des années 1990. À partir de ce moment, la Banque mondiale a commencé à revoir sa stratégie. Elle est de moins en moins une banque qui prête et qui attend des remboursements et des dividendes, mais se transforme en «banque de savoir œuvrant en partenariat avec les donateurs, les clients et les organisations » (traduction) ${ }^{12}$. Quant aux deux autres bailleurs de fonds, l'évolution du cadre institutionnel et la gestion plus orientée vers les résultats et le retour en force de l'aide budgétaire ont facilité la mise en œuvre de l'évaluation participative. L'évaluation participative des institutions est donc en perpétuelle mutation et comme nous venons de le démontrer, il n'existe pas de modèle unique et unanimement reconnu en la matière, et ce, malgré l'existence de repères théoriques en la matière.

Ces conclusions pourraient être affinées en étudiant en profondeur le processus évaluatif à l'aide notamment d'études de cas ou de métaévaluations (évaluation des évaluations).

2 Jackson, E. T., et Y. Kassam (1998). "Introduction" dans E. T. Jackson et Y. Kassam (dirs.). Knowledge Shared: Participatory Evaluation in Development Cooperation, Ottawa / West Hartford, CT : International Development Research Centre / Kumarian Press, (p. 116).

3 Cousins, J. B. et E. Whitmore (1998). Framing Participatory Evaluation. New Directions for Evaluation vol. 80, pp. 5-23; Weaver, L., et J. B. Cousins (2004). Unpacking the Participatory Process. Journal of MultiDisciplinary Evaluation, (1), pp. 19-40.

4 Floc'hlay, B et E. Plottu (1998). Participation et décision publique : De l'incantation à la mise en œuvre d'une évaluation démocratique. Revue canadienne d'évaluation de programme, vol. 13 (1), pp. 39-60; 
Fricke, J. et R. Gill. (1989). Participative Evaluation. The Canadian Journal of Program Evaluation, vol. 4 (1), pp. 11-25.

5 Bamberger, M. (2000). The Evaluation of International Development Programs: A View from the Front. American Journal of Evaluation, vol. 21 (1), pp. 95-102; Bamberger, M. (2001). Book Review: Evaluation in Developing Countries: Experience with Agricultural Research and Development. The Annotated Bibliography of International Program Evaluation. American Journal of Evaluation, vol. 22 (1), pp. 117-122.

6 Guba, E. G. et Y. S. Lincoln (1989). Fourth Generation Evaluation. London : Sage; Lawrence, G. (1989). Engaging Recipients in Development Evaluation: The Stakeholders Approach. Evaluation Review, vol.13 (3), pp. 243-256;

Patton, M. Q. (1997). Utilization-Focused Evaluation, $3^{\mathrm{e}}$ éd, London : Sage; Pawson, R. et N. Tilley (1997).

Realistic Evaluation. London : Sage; Rebien, C. C. (1996). Participatory Evaluation of Development Assistance: Dealing with Power and Facilitative Learning. Evaluation, 2 (2), pp. 151-172; Ridde, V. (2006). Suggestion d'améliorations d'un cadre conceptuel de l'évaluation participative. Revue canadienne d'évaluation de programme, vol. 21 (2), pp. 1-23;

Rifkin S.B., Muller, F. et W. Bichmann, (1988). Primary Health Care: On Measuring Participation. Social Science Medicine, vol. 9, pp. 931-940; Rugh, J. (1986). SelfEvaluation: Ideas for Participatory Evaluation of Rural Development Projects; Oklahoma City, OK: World Neighbours; Sabatier, P. A. et H. C. Jenkins-Smith (1993). Policy Change and Learning: An Advocacy Coalition Approach. Boulder, CO: Westview Press;
Smith, N. L. (2002). International Students' Reflections on the Cultural Embeddedness of Evaluation Theory. American Journal of Evaluation, vol. 23 (4), pp. 481-492;

7 Dowing, K. M. (1996). Power, Minneapolis : University of Minnesota Press; Daigneault P.-M. et S. Jacob (2009). « Toward Accurate Measurement of Participation: Rethinking the Conceptualization and Operationalization of Participatory Evaluation », American Journal of Evaluation, vol. 30, no 3, pp. 330-348; European Commission (2004). Evaluating EU Activities: A Practical Guide for the Commission Services, http://ec.europa.eu/europeaid/who/index fr.htm, Bruxelles: EC, [consulté le 21 novembre 2007]; Fetterman, D. M. (2001). Foundations of Empowerment Evaluation, Thousand Oaks, Sage.

8 OED, (2004). Suivi et évaluation : Quelques outils, méthodes et approches, Washington DC : Banque mondiale.

9 Agence canadienne de développement international. Direction de l'évaluation. Direction générale de la gestion du rendement et des Connaissances (2004). Guide de l'évaluation, Gatineau, QC : ACDI, http://www.acdi-cida.gc.ca/cidaweb/acdicida.nsf/Fr/NIC -5313423-N2A, [consulté le 19 novembre 2007].

${ }^{10}$ http://ec.europa.eu/europeaid/who/index fr.htm [consulté le 21 novembre 2007].

${ }^{11}$ Lefebvre, L et I., Yong, (2006). L'évaluation des programmes d'aide au développement conduits par la Commission européenne. Les grandes synthèses thématiques et par pays, Télescope, vol.13 (1), pp. 87-103.

12 McAllister, E. (2006). OED Renewal: Participation, Partnership, and Results. 